\title{
Holomorphic Curves in Loop Groups
}

\author{
M. A. Guest ${ }^{1}$ and A. N. Pressley ${ }^{2}$ \\ ${ }^{1}$ Department of Mathematics, University of Rochester, NY 14627, USA \\ ${ }^{2}$ Department of Mathematics, Kings College, Strand, London WC2R 2LS, UK
}

\begin{abstract}
It was observed by Atiyah that there is a correspondence between based gauge equivalence classes of $S U_{n}$-instantons over $S^{4}$ of charge $d$ on the one hand, and based holomorphic curves of genus zero in $\Omega S U_{n}$ of degree $d$ on the other hand. In this paper we study the parameter space of such holomorphic curves which have the additional property that they lie entirely in the subgroup $\Omega_{\mathrm{alg}} S U_{n}$ of algebraic loops. We describe a cell decomposition of this parameter space, and compute its complex dimension to be $(2 n-1) d$.
\end{abstract}

\section{Introduction}

It is well known that the space $\Omega G$ of (smooth) basepoint preserving maps from the circle $S^{1}$ to a compact Lie group $G$ is, in a natural way, a complex manifold. One of its many remarkable properties is that, despite being infinite dimensional, $\Omega G$ behaves in many ways as if it were a compact manifold. For example, every holomorphic function $\Omega G \rightarrow \mathbb{C}$ is constant. Atiyah [At] proved that, for any compact, complex manifold $M$, the set of all basepoint preserving holomorphic maps $M \rightarrow \Omega G$ lying in a given homotopy class is finite dimensional; in simple cases, the dimension can even be computed. The argument in [At], however, is non-constructive. The purpose of this paper is to complement [At] by giving an explicit geometric construction of a large family of holomorphic maps $M \rightarrow \Omega G$ in the case where $M$ is the Riemann sphere $S^{2}$. Some examples of where the study of holomorphic maps $M \rightarrow \Omega G$ occurs in the literature are given at the end of the introduction.

To describe our results more precisely, we assume, without loss of generality, that $G$ has only one simple factor, so that $\pi_{2}(\Omega G) \cong \pi_{3}(G) \cong \mathbb{Z}$. Then any (continuous) map $S^{2} \rightarrow \Omega G$ has an integer invariant, its degree, given by the induced map on $\pi_{2}$, and this determines the map up to homotopy. Let $\operatorname{Hol}_{d}^{*}\left(S^{2}, \Omega G\right)$ denote the set of holomorphic maps $f: S^{2} \rightarrow \Omega G$ of degree $d$, which are basepoint preserving in the sense that $f(\infty)=e$, where we think of $S^{2}=\mathbb{C} \cup\{\infty\}$ as the extended complex plane and $e$ is the identity element or identity loop in $G$. Here, $d$ is necessarily $\geqq 0$. Then $[\mathrm{At}]$ gives: 
(1.1) $\mathrm{Hol}_{d}^{*}\left(S^{2}, \Omega G\right)$ is a complex manifold of dimension $2 d c(G)$, where $c(G)$ is the dual Coxeter number of $G$.

For example, if $G=S U_{n}$, the dimension is $2 n d$ for $n \geqq 2$.

Our construction of holomorphic maps $S^{2} \rightarrow \Omega G$ makes use of the subgroup $\Omega_{\text {alg }} G$ of $\Omega G$ consisting of the maps $S^{1} \rightarrow G$ which have finite Fourier expansions. Although this is not a submanifold, it is a good approximation to $\Omega G$ in at least two senses (see [PS]):

(1.2) $\Omega_{\mathrm{alg}} G$ is homotopy equivalent to $\Omega G$;

(1.3) $\Omega_{\mathrm{alg}} G$ is dense in $\Omega G$, if $G$ is semisimple.

An infinite dimensional version of Chow's theorem would suggest that every based holomorphic map $S^{2} \rightarrow \Omega G$ actually maps into $\Omega_{\mathrm{alg}} G$. The results of this paper, however, indicate that this is probably false.

We shall make use of a filtration

$$
\{I\}=\Omega_{0} \subset \Omega_{1} \subset \Omega_{2} \subset \ldots \subset \Omega_{\mathrm{alg}} G=\bigcup_{k=0}^{\infty} \Omega_{k}
$$

of $\Omega_{\mathrm{alg}} G$ by (finite dimensional) complex projective varieties $\Omega_{k}$. In fact, the $\Omega_{k}$ are varieties of fixed points of unipotent transformations acting on Grassmannians; such varieties have been studied extensively in [Sh, HS, Bo]. We shall show that $\operatorname{Hol}_{d}^{*}\left(S^{2}, \Omega_{k}\right)$ is essentially an iterated fibration whose base and fibres are varieties of this type. We set

$$
\operatorname{Hol}_{d}^{*}\left(S^{2}, \Omega_{\mathrm{alg}} S U_{n}\right)=\bigcup_{k=0}^{\infty} \operatorname{Hol}_{d}^{*}\left(S^{2}, \Omega_{k}\right)
$$

we conjecture that every based holomorphic map $S^{2} \rightarrow \Omega G$ with values in $\Omega_{\text {alg }} G$ actually maps into some $\Omega_{k}$, so that the notation should be unambiguous. One consequence of our description of $\operatorname{Hol}_{d}^{*}\left(S^{2}, \Omega_{\mathrm{alg}} S U_{n}\right)$, to be proved in Sect. 4, is:

Corollary 1.4. $\mathrm{Hol}_{d}^{*}\left(S^{2}, \Omega_{\mathrm{alg}} S U_{n}\right.$ ) has (Hausdorff) dimension $2(2 n-1) d$. (We use Hausdorff dimension as $\mathrm{Hol}_{d}^{*}\left(S^{2}, \Omega_{\mathrm{alg}} S U_{n}\right)$ is not a manifold.)

Comparing with (1.1), we see that there are based holomorphic maps $S^{2} \rightarrow \Omega S U_{n}$ which do not map into any $\Omega_{k}$. One can show that every based holomorphic map $M \rightarrow \Omega G$, for any compact complex manifold $M$, actually maps into $\Omega_{\text {rat }} G$, the rational functions $S^{1} \rightarrow G$, but the methods of this paper (based on those of $[\mathrm{Gu}]$ ) do not apply to $\Omega_{\mathrm{rat}} G$.

The simplest non-trivial case of (1.4), namely $d=1, n=2$, is easy to understand geometrically. We shall see that $\operatorname{Hol}_{1}^{*}\left(S^{2}, \Omega_{\mathrm{alg}} S U_{2}\right)=\operatorname{Hol}_{1}^{*}\left(S^{2}, \Omega_{1}\right)$, and that $\Omega_{1}$ is a cone on a conic in $\mathbb{C} P^{2}$ (see Example 3.2 for a more precise statement) whose vertex is the identity loop $I$ (see Fig. 1). The basepoint preserving degree 1 maps $S^{2} \rightarrow \Omega_{1}$ are given by the generators of the cone, a complex 1-parameter family. But each generator has a complex 2-parameter family of parametrisations which respect basepoints. Thus, $\operatorname{Hol}_{1}^{*}\left(S^{2}, \Omega_{\mathrm{alg}} S U_{2}\right)$ is 6-dimensional whereas, by (1.1), $\mathrm{Hol}_{1}^{*}\left(\mathrm{~S}^{2}, \Omega S U_{2}\right)$ is 8 dimensional.

We conclude this introduction by mentioning some problems in the calculus of variations where holomorphic maps of a compact Riemann surface $M$ into $\Omega G$ have arisen naturally. 
Fig. 1

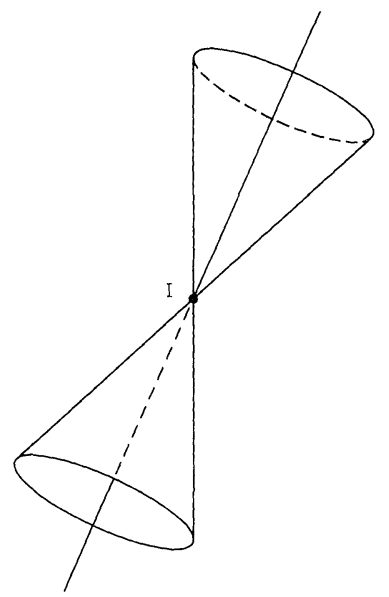

\section{Example A: Topology of the Space of Holomorphic Curves}

The loop group $\Omega G$ is not only a complex manifold but also a homogeneous Kähler manifold [PS], which has many properties in common with finite dimensional homogeneous Kähler manifolds such as projective spaces or Grassmannians. Segal $[\mathrm{Se}]$ proved that $\operatorname{Hol}_{d}^{*}\left(M, \mathbb{C P}^{n}\right)$ approximates the space $\operatorname{Map}_{d}^{*}\left(M, \mathbb{C P}^{n}\right)$ of smooth (or continuous) maps:

(1.5) The inclusion $\operatorname{Hol}_{d}^{*}\left(M, \mathbb{C} P^{n}\right) \hookrightarrow \operatorname{Map}_{d}^{*}\left(M, \mathbb{C} P^{n}\right)$ is a homology equivalence up to dimension $(d-2 g)(2 n-1)$, where $g$ is the genus of $M$. Moreover, if $g=0\left(M=S^{2}\right)$, the inclusion is a homotopy equivalence in the same range.

Although Segal's proof of (1.5) was entirely topological, the result has an interesting interpretation in terms of differential geometry, since $\operatorname{Hol}_{d}^{*}\left(M, \mathbb{C} P^{n}\right)$ is precisely the set of absolute minima of the energy functional $E: \operatorname{Map}_{d}^{*}\left(M, \mathbb{C} P^{n}\right) \rightarrow \mathbb{R}$ given by

$$
E(f)=\int_{M}\|d f\|^{2} .
$$

Thus, (1.5) should admit a Morse-theoretic interpretation.

These results were partially generalized to the case where $\mathbb{C} P^{n}$ is replaced by a Grassmannian or flag manifold in [Gu, Ki]. It was conjectured by Atiyah [At] that similar results should hold when $\mathbb{C} P^{n}$ is replaced by $\Omega G$; some progress has been made in this direction (see Example B).

\section{Example B: Holomorphic Curves and Yang-Mills Instantons}

Let $P$ be a principal $G$-bundle on $S^{4}$. Since $\pi_{3}(G) \cong \mathbb{Z}, P$ is determined up to topological equivalence by an integer $d$. Assume $d \geqq 0$. Let $\mathscr{A}_{d}$ be the space of connections on $P$ and $\mathscr{I}_{d}$ the space of self-dual connections (instantons). The group $\mathscr{G}_{*}$ of automorphisms of $P$ which are the identity in the fibre over a fixed basepoint of $S^{4}$ acts freely on $\mathscr{A}_{d}$. In [At], Atiyah proved that there is a diffeomorphism $\mathscr{I}_{d} / \mathscr{G}_{*} \cong \operatorname{Hol}_{d}^{*}\left(S^{2}, \Omega G\right)$, for any classical $G$. Combining this with the results of [AHS] gives (1.1) immediately. 
We can now relate the conjecture in Example A to earlier work of Atiyah and Jones $[\mathrm{AJ}]$. Consider the following diagram:

$$
\begin{gathered}
\mathscr{I}_{d} / \mathscr{G}_{*} \rightarrow \operatorname{Hol}_{d}^{*}\left(S^{2}, \Omega G\right) \\
\downarrow \\
\mathscr{A}_{d} / \mathscr{G}_{*} \rightarrow \operatorname{Map}_{d}^{*}\left(S^{2}, \Omega G\right)
\end{gathered}
$$

The lower horizontal map is a homotopy equivalence, described in [AJ]. For $G=S U_{2}$, it was proved in [AJ] that the left-hand inclusion is, in homology, a projection onto a direct summand in dimensions $\ll d$. It was conjectured that one actually obtains a homotopy equivalence in dimensions $\ll d$; hence the same would hold for the right-hand inclusion. A version of this conjecture, i.e.

$$
\lim _{d \rightarrow \infty} H_{*} \operatorname{Hol}_{d}^{*}\left(S^{2}, \Omega G\right)=\lim _{d \rightarrow \infty} H_{*} \operatorname{Map}_{d}^{*}\left(S^{2}, \Omega G\right)
$$

has been proved by Graveson [Gv]. Using the instanton formulation this has also been proved by Taubes [Ta]. See also [BM] and the Note added in proof at the end of this paper.

In the case $G=S U_{2}, d=1$, instantons are well understood (see Sect. 6 of [FU]): $\mathscr{I}_{1} / \mathscr{G}_{*}\left(\cong \operatorname{Hol}_{1}^{*}\left(S^{2}, \Omega S U_{2}\right)\right)$ is the real 8 dimensional manifold $S O_{3} \times B^{5}$, where $B^{5}$ is the open 5-ball. We do not know whether the real 6 dimensional manifold corresponding to $\mathrm{Hol}_{1}^{*}\left(S^{2}, \Omega_{\mathrm{alg}} S U_{2}\right)$ has any significance in terms of instantons [the diffeomorphism $\mathscr{I}_{d} / \mathscr{G}_{*} \cong \mathrm{Hol}_{d}^{*}\left(S^{2}, \Omega G\right)$ is not canonical]. However, from the identification $\mathrm{Hol}_{1}^{*}\left(S^{2}, \Omega_{\text {alg }} S U_{2}\right) \cong P S L_{2}(\mathbb{C})$ made at the end of Sect. 4, it follows that this 6 dimensional manifold is diffeomorphic to $\mathrm{SO}_{3} \times B^{3}$.

\section{Example C: Classical Solutions of the Principal Chiral Model}

The principal chiral model is a field theory which, at the classical level, amounts to studying harmonic maps $M \rightarrow G$, where $M$ is a compact Riemann surface. In [Uh] (see also $[\mathrm{ZM}, \mathrm{ZS}]$ ) it was proved that there is a one-to-one correspondence between the set of based harmonic maps $\phi: S^{2} \rightarrow G$ and the set of "extended solutions", i.e., the set of maps $\Phi: S^{2} \rightarrow \Omega G$ satisfying

(1) $\Phi(\infty)=e$,

(2) $(\partial \Phi / \partial \lambda) \Phi^{-1}=\left(1-z^{-1}\right) A$, and

(3) $(\partial \Phi / \partial \bar{\lambda}) \Phi^{-1}=(1-z) B$,

where $A, B$ are functions on $S^{2}$ with values in the Lie algebra of $G$ and $z$ is the loop parameter in $\Omega G$. Moreover, the degree of $\Phi$ is the (suitably normalized) energy of $\phi$ (this is an observation of Eells and Freed). Hence, a crude upper bound for the real dimension of the space of based harmonic maps $S^{2} \rightarrow G$ of energy $d$ is [by (1.1)] $4 d c(G)$. A theorem of [Uh] says that, for $G=U_{n}$, any extended solution actually takes value in some $\Omega_{k}$, so our result permits a slight reduction of this upper bound, from 4 nd to $2(2 n-1) d$.

\section{Aspects of the Geometry of $\Omega G$}

In this section we summarize the necessary facts about loop groups. Our main reference is $[\mathrm{PS}]$, although $[\mathrm{Pr}]$ contains most of the information we need. 
For any Lie group $G$, the loop group $L G$ is the space of smooth maps $S^{1} \rightarrow G$, provided with the $C^{\infty}$-topology; $L G$ is a Fréchet Lie group. The based loop group $\Omega G$ is the space of maps $f: S^{1} \rightarrow G$ satisfying $f(1)=e$. Although $\Omega G$ is a subgroup of $L G$, it is better to think of $\Omega G$ as a homogeneous space of $L G$ :

$$
\Omega G \cong L G / G,
$$

where $G$ is identified with the constant loops in $L G$. By way of contrast, the groups $L_{\mathrm{alg}} G$ and $\Omega_{\mathrm{alg}} G$, consisting of maps $S^{1} \rightarrow G$ which have finite Fourier expansions, are not smooth manifolds in any reasonable sense. (For us, $G$ will always be a matrix group, so the definition of $L_{\mathrm{alg}} G$ is obvious; in general, one would have to take linear representations of $G$.)

All the geometry of $\Omega G$ which we shall need can be deduced from the "Grassmannian model" of $\Omega G$. We shall describe this only when $G=U_{n}$ or $S U_{n}$, although the model exists for all compact $G$. Let $H$ be the Hilbert space $L^{2}\left(S^{1}, \mathbb{C}^{n}\right)$, $n \geqq 1$. Then $\Omega U_{n}$ acts on $H$ (by unitary operators) and hence also on the subspaces of $H$. Let $H_{+} \subseteq H$ be the closed subspace of $H$ consisting of the vectors $v \in H$ of the form

$$
v=\sum_{j \geqq 0} v_{j} z^{j}, \quad v_{j} \in \mathbb{C}^{n}
$$

where $S^{1}$ is thought of as the set of complex numbers $z$ of modulus 1 .

Theorem 2.1 (Grassmannian Model). The assignment $f \mapsto f H_{+}$is a one-to-one correspondence between $\Omega_{\mathrm{alg}} U_{n}$ and the set of subspaces $W$ of $H$ such that

(i) $z W \subseteq W$, and

(ii) $z^{k} H_{+} \subseteq W \leqq z^{-k} H_{+}$for some $k \geqq 0$.

The group $\Omega_{\text {alg }} U_{n}$ has components indexed by $\mathbb{Z}$ : a loop $f$ lies in the $d$-th component if $\operatorname{det} f: S^{1} \rightarrow S^{1}$ has winding number $-d$. In terms of the Grassmannian model,

$$
d=\frac{1}{2}\left(\operatorname{dim}\left(W / z^{k} H_{+}\right)-\operatorname{dim}\left(z^{-k} H_{+} / W\right)\right),
$$

where $W=f H_{+}$and $k$ is any integer large enough for (2.1)(ii) to hold. Since any trigonometric polynomial map $S^{1} \rightarrow S^{1}$ of degree zero is constant, $\Omega_{\text {alg }} S U_{n}$ is exactly the identity component of $\Omega_{\text {alg }} U_{n}$. The larger group $L_{\text {alg }} S L_{n}(\mathbb{C})$ obviously acts on the set of subspaces satisfying (2.1) (i) and (ii), and the stabilizer of $H_{+}$is the subgroup $L_{\text {alg }}^{+} S L_{n}(\mathbb{C})$ consisting of the loops which involve no negative powers of $z$. Thus we have a one-to-one correspondence

$$
L_{\mathrm{alg}} S L_{n}(\mathbb{C}) / L_{\mathrm{alg}}^{+} S L_{n}(\mathbb{C}) \cong \Omega_{\mathrm{alg}} S U_{n}
$$

There is also a Grassmannian model for the smooth loop group $\Omega U_{n}$ : one replaces (2.1)(ii) by the condition that the images of the orthogonal projections $W \rightarrow H_{+}^{\perp}$ and $W^{\perp} \rightarrow H_{+}$should consist of smooth maps. In particular, the smooth analogue of (2.3) holds, in the sense that there is a diffeomorphism

$$
L S L_{n}(\mathbb{C}) / L^{+} S L_{n}(\mathbb{C}) \cong \Omega S U_{n} .
$$

Since $L S L_{n}(\mathbb{C})$ and $L^{+} S L_{n}(\mathbb{C})$ are complex Lie groups, this shows that $\Omega S U_{n}$ is a complex manifold. 
Theorem 2.1 immediately suggests a filtration of $\Omega_{\mathrm{alg}} S U_{n}$ (or $\left.\Omega_{\mathrm{alg}} U_{n}\right)$. Namely, let $\Omega_{k}$ denote the set of loops $f \in \Omega_{\text {alg }} S U_{n}$ for which $W=f H_{+}$satisfies (2.1) (ii) for the given value of $k$. Then

$$
\Omega_{0} \subset \Omega_{1} \subset \Omega_{2} \subset \ldots \subset \Omega_{\mathrm{alg}} S U_{n}=\bigcup_{k \geqq 0} \Omega_{k} .
$$

In terms of loops, $\Omega_{k}$ consists of those $f \in \Omega_{\mathrm{alg}} S U_{n}$ of the form

$$
f(z)=\sum_{j=-k}^{k} A_{j} z^{j}
$$

for some $n \times n$ matrices $A_{j}$. Note that $\Omega_{0}$ consists of a single point, the constant loop in $\Omega_{\mathrm{alg}} S U_{n}$. In general, Theorem 2.1 shows that $\Omega_{k}$ is a complex projective variety. To see this, observe first that multiplication by $z$ is a nilpotent endomorphism of $z^{-k} H_{+} / z^{k} H_{+}$. Using (2.2), it follows that the assignment $f \mapsto f H_{+} / z^{k} H_{+}$identifies $\Omega_{k}$ with the set of $X \in \mathrm{Gr}_{k n}\left(z^{-k} H_{+} / z^{k} H_{+}\right)$, the Grassmannian of $k n$ dimensional subspaces of the $2 k n$ dimensional space $z^{-k} H_{+} / z^{k} H_{+}$, for which $z X \subseteq X$; equivalently, $X$ should be fixed by the unipotent automorphism $1+z$. It follows that $\Omega_{k}$ is a (closed) subvariety of $\mathrm{Gr}_{k n}\left(z^{-k} H_{+} / z^{k} H_{+}\right) \cong \mathrm{Gr}_{k n}\left(\mathbb{C}^{2 k n}\right)$. Except for $\Omega_{0}$, all the $\Omega_{k}$ are singular varieties.

It is casy to see that the natural complex structure and topology on the variety $\Omega_{k}$ agree with those which are inherited from $\Omega S U_{n}$. However, the topology on $\Omega_{\text {alg }} S U_{n}$ induced from the $C^{\infty}$-topology on $\Omega S U_{n}$ is strictly coarser than the direct limit topology arising from the identification $\Omega_{\mathrm{alg}} S U_{n}=\bigcup_{k \geqq 0} \Omega_{k}$.

Finally, it can be shown that $\Omega_{\text {alg }} S U_{n}$ has a natural cell decomposition arising from the Grassmannian model, and that $\Omega_{k}$ consists precisely of the $k n^{2}$-skeleton (if $n$ is even) or the $k\left(n^{2}-1\right)$-skeleton (if $n$ is odd) for this decomposition. Together with (1.2), this implies that the inclusions $\Omega_{k} \hookrightarrow \Omega_{\text {alg }} S U_{n} \hookrightarrow \Omega S U_{n}$ induce isomorphisms on $\pi_{2}$ (for $k \geqq 1, n \geqq 2$ ) and so the notation $\operatorname{Hol}_{d}^{*}\left(S^{2}, \Omega_{k}\right)$ makes sense.

\section{Fixed Point Varieties of Unipotent Transformations}

Let $F=F\left(r_{1}, \ldots, r_{s}\right)$ be the complex "flag manifold" consisting of all sequences $\left\{E_{i}\right\}$ of the form

$$
\{0\} \subset E_{r_{1}} \subset E_{r_{2}} \subset \ldots \subset E_{r_{s}}=\mathbb{C}^{n},
$$

where $E_{i}$ is a subspace of $\mathbb{C}^{n}$ of dimension $i$. Let $N=N\left(n_{1}, \ldots, n_{m}\right)$ be the nilpotent linear transformation of $\mathbb{C}^{n}$ which is the direct sum of Jordan blocks $J_{n_{1}}, \ldots, J_{n_{m}}$, where $J_{l}$ is represented by the $i \times i$ matrix

$$
\left(\begin{array}{rrrrr}
0 & 1 & & \\
& \cdot & & \\
& & \cdot & \cdot & 1 \\
& & & 0
\end{array}\right)
$$

with respect to some fixed basis. Then we are interested in

$$
F^{N}=\left\{\left\{E_{i}\right\} \in F \mid N E_{i} \subseteq E_{i} \text { for all } i\right\},
$$


which is the same as the set of fixed points in $F$ of the unipotent transformation $U=I+N$. The case of the "full flag manifold" [i.e. $F=F(1,2, \ldots, n)]$ has been studied in greater generality, namely, where $F$ is replaced by the quotient of a complex semisimple Lie group by a Borel subgroup, and where $N$ is replaced by any nilpotent element of the Lie algebra. The space $F^{N}$ has many interesting properties. For example, it provides a link between nilpotent orbits in the Lie algebra and representations of the Weyl group: to the orbit of $N$ one associates a certain action of the Weyl group on (a subgroup of) the top cohomology group of $F^{N}$. For a summary of this, and further references, see [Bo].

The variety $\Omega_{k}$ of Sect. 2 is isomorphic to $\mathrm{Gr}_{k n}\left(\mathbb{C}^{2 k n}\right)^{N(2 k, \ldots, 2 k)}$, and we identify these from now on. Thus, all we need are some facts concerning $F^{N}$ in the special case $s=2$, which we shall derive from scratch.

The main result is the following, which we take from [Sh]. (A more general reference, which uses slightly different conventions, is [HS].)

Theorem 3.1. The space $\mathrm{Gr}_{k}\left(\mathbb{C}^{n}\right)^{N}$ (where $N=N\left(n_{1}, \ldots, n_{m}\right)$ ) has a complex cell decomposition, the cells being indexed by diagrams

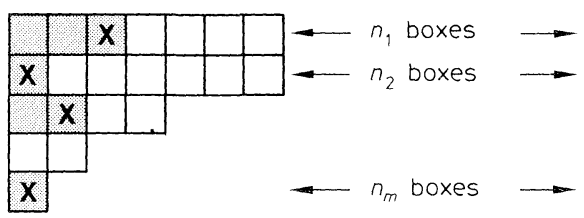

in which crosses are distributed in such a way that

(1) now row contains more than one cross, and

(2) if the boxes in each row to the left of (and including) the crosses are shaded, there are $k$ shaded boxes altogether.

We define an ordering on the boxes in such a diagram, by saying that one box precedes another of the first box is either in a column to the left of, or in the same column and above, the second box.

The complex dimension of the cell corresponding to such a diagram is $\Sigma d_{i}$, where $d_{i}$ is the number of unshaded boxes which precede the box containing the $i$-th cross. (In the diagram shown, $\Sigma d_{i}=6$ ). If $X$ belongs to the cell corresponding to the diagram with $k_{i}$ shaded boxes in row $i\left(0 \leqq k_{i} \leqq n_{i}\right)$, then the restriction of $N$ to $X$ is nilpotent and has type given by $N\left(k_{1}, \ldots, k_{m}\right)$.

Proof. First we recall the usual cell decomposition of $\mathrm{Gr}_{k}\left(\mathbb{C}^{n}\right)$. Let $e_{1}, \ldots, e_{n}$ be an orthonormal basis of $\mathbb{C}^{n}$, and let $v_{1}, \ldots, v_{k}$ be a basis for $V \in \mathrm{Gr}_{k}\left(\mathbb{C}^{n}\right)$. We write $\mathbb{C}^{n}=\left[e_{1}, \ldots, e_{n}\right], V=\left[v_{1}, \ldots, v_{k}\right]$, and $v_{i}=\sum_{j=1}^{n} a_{i j} e_{j}, j=1, \ldots, k$. The $k \times n$ matrix $A=\left(a_{i j}\right)$ may be put in reduced echelon form. In other words, there exists an invertible $k \times k$ matrix $R$ such that $R A$ has the form:

$$
\left(\begin{array}{llllllll}
* & * & 1 & 0 & 0 & 0 & 0 & 0 \\
* & * & 0 & 1 & 0 & 0 & 0 & 0 \\
* & * & 0 & 0 & * & 1 & 0 & 0 \\
* & * & 0 & 0 & * & 0 & 1 & 0
\end{array}\right)
$$


Let the 1's appear in columns $s_{1}, \ldots, s_{k}$ (where $1 \leqq s_{1}<\ldots<s_{k} \leqq n$ ). This matrix, and hence the set $S_{V}=\left\{s_{1}, \ldots, s_{k}\right\}$, is canonically assigned to the subspace $V$. Since the $*$ 's are unrestricted, it follows, given any set $S=\left\{s_{1}, \ldots, s_{k}\right\} \subseteq\{1,2, \ldots, n\}$ with $s_{1}<\ldots<s_{k}$, that the set

$$
c_{S}=\left\{V \in \mathrm{Gr}_{k}\left(\mathbb{C}^{n}\right) \mid S_{V}=S\right\}
$$

is a cell, i.e., a submanifold of $\mathrm{Gr}_{k}\left(\mathbb{C}^{n}\right)$ diffeomorphic to $\mathbb{C}^{d}$ for some $d$. The dimension $d$ is given simply by counting the number of entries $*$, so

$$
d=\sum_{i=1}^{k}\left(s_{i}-i\right)
$$

Thus, $\mathrm{Gr}_{k}\left(\mathbb{C}^{n}\right)=\cup c_{S}$, where the cells $c_{S}$ are indexed by matrices of the form

$$
\left(\begin{array}{llllllll}
0 & 0 & 1 & 0 & 0 & 0 & 0 & 0 \\
0 & 0 & 0 & 1 & 0 & 0 & 0 & 0 \\
0 & 0 & 0 & 0 & 0 & 1 & 0 & 0 \\
0 & 0 & 0 & 0 & 0 & 0 & 1 & 0
\end{array}\right)
$$

which have $k$ entries equal to 1 , not more than one such in each row and column, and with all other entries zero. We may compress this information into a diagram of the form

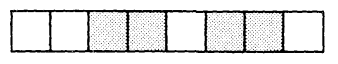

with precisely $k$ shaded boxes, from which the dimension $d$ is easily read off.

The same method may be used to give a cell decomposition of $\mathrm{Gr}_{k}\left(\mathbb{C}^{n}\right)^{N}$. Let $\mathbb{C}^{n}=V_{1} \oplus \ldots \oplus V_{m}$, where the restriction of $N$ to $V_{i}$ is cyclic, i.e., represented by a Jordan block $J_{n_{1}}$ with respect to some basis. Let the basis $e_{1}, \ldots, e_{n}$ of $\mathbb{C}^{n}$ be obtained by taking the union of such bases for $V_{1}, \ldots, V_{m}$. In the following diagram (which generalizes the one above) we arrange the boxes so that the $i$-th row represents the chosen basis of $V_{i}$ :

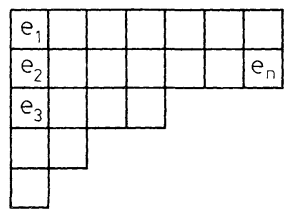

The action of $N$ on basis vectors is thus represented by a shift of one box (to the left, say). The first $j$ columns together represent $\operatorname{Ker} N^{j}$. For $S=\left\{s_{1}, \ldots, s_{k}\right\}$, we call an element $\left[e_{s_{1}}, \ldots, e_{s_{k}}\right] \in \mathrm{Gr}_{k}\left(\mathbb{C}^{n}\right)$ a "standard $k$-plane"; if $\left[e_{s_{1}}, \ldots, e_{s_{k}}\right] \in \mathrm{Gr}_{k}\left(\mathbb{C}^{n}\right)^{N}$ we call it a "standard invariant $k$-plane." The standard $k$-planes are represented on the diagram by shading $k$ boxes, and these index the cells of $\mathrm{Gr}_{k}\left(\mathbb{C}^{n}\right)$. The standard invariant $k$-planes are represented by shading $k$ boxes in such a way that $N$ always moves a shaded box to another shaded box, for example: 


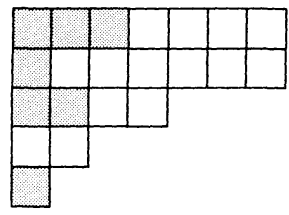

The cell $c_{S}$ of $\mathrm{Gr}_{k}\left(\mathbb{C}^{n}\right)$ consists of the standard $k$-plane $V_{S}=\left[e_{s_{1}}, \ldots, e_{s_{k}}\right]$ together with all "modifications" of it, where a modification involves altering the appropriate $s_{i}-i$ coordinates of the basis vectors $e_{s_{i}}$. If $V_{S} \in \mathrm{Gr}_{k}\left(\mathbb{C}^{n}\right)^{N}$, a subset $c_{S}^{N} \cong \mathrm{Gr}_{k}\left(\mathbb{C}^{n}\right)^{N} \cap c_{S}$ is obtained by modifying $\left[e_{s_{1}}, \ldots, e_{s_{k}}\right]$ to $\left[v_{s_{1}}, \ldots, v_{s_{k}}\right]$, where $N v_{i}=v_{j}$ if and only if $N e_{i}=e_{j}$. Such modifications are specified by making alterations (of the kind just described) to the basis vectors at the right-hand ends of each row of shaded boxes. Such boxes we indicate with a cross:

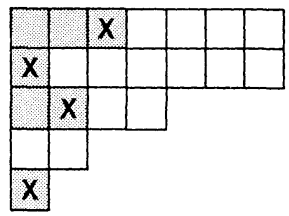

Thus, $c_{S}^{N}$ is a cell, and its dimension is $\sum d_{i}$, where $d_{i}$ is the number of unshaded boxes "preceding" the $i$-th cross (the ordering being that of the basis vectors $e_{1}, \ldots, e_{n}$ ). For example, in particular diagram above, we have a cell in $\operatorname{Gr}_{7}\left(\mathbb{C}^{21}\right)^{N}$ of dimension $d_{1}+d_{2}+d_{3}+d_{4}=0+1+2+3=6$. The fact that the restriction of $N$ to $X$ is $N\left(k_{1}, \ldots, k_{m}\right)$, for any $X$ in this cell, is obvious. To complete the proof of the theorem, we show that $c_{S}^{N}$ is in fact equal to $\mathrm{Gr}_{k}\left(\mathbb{C}^{n}\right)^{N} \cap c_{S}$. First, it is easy to see that $\mathrm{Gr}_{k}\left(\mathbb{C}^{n}\right)^{N} \cap c_{S} \neq \emptyset$ if and only if $V_{S} \in \mathrm{Gr}_{k}\left(\mathbb{C}^{n}\right)^{N}$. When this is so, the reduced echelon form shows that any $W \in \mathrm{Gr}_{k}\left(\mathbb{C}^{n}\right)^{N} \cap c_{S}$ has a basis $w_{s_{1}}, \ldots, w_{s_{k}}$ with the property: $N w_{s_{t}}=w_{s_{j}}$ if and only if $N e_{s_{t}}=e_{s_{j}}$. Hence $W$ belongs to $c_{S}^{N}$.

Example 3.2. The Grassmannian $\mathrm{Gr}_{2}\left(\mathbb{C}^{4}\right)$ has 6 cells represented by:
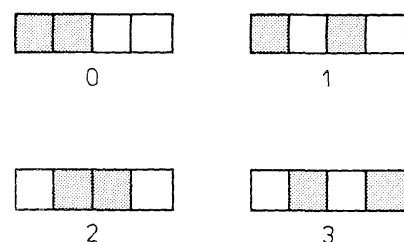

3
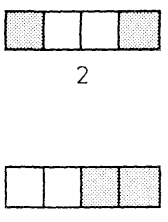

4

where the dimensions are as indicated. If $N=N(2,2)$, the corresponding cells of $\mathrm{Gr}_{2}\left(\mathbb{C}^{4}\right)^{N}$ are represented by:

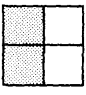

0

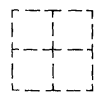

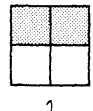

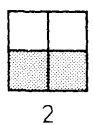

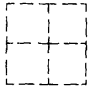

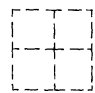


If $\mathrm{Gr}_{2}\left(\mathbb{C}^{4}\right)$ is represented in the usual way as a quadric in $\mathbb{C} P^{5}$ given by the Plücker equation $z_{0} z_{1}-z_{2} z_{3}+z_{4} z_{5}=0$, an elementary calculation shows that $\Omega_{1}\left(=\operatorname{Gr}_{2}\left(\mathbb{C}^{4}\right)^{N(2,2)}\right)$ corresponds to the subvariety given by imposing the additional conditions $z_{0}=0, z_{4}=z_{5}$. Hence,

$$
\Omega_{1} \cong\left\{\left[z_{0} ; z_{1} ; z_{2} ; z_{3}\right] \in \mathbb{C} P^{3} \mid z_{1} z_{2}=z_{3}^{2}\right\} \text {. }
$$

Blowing up the singular point $[1 ; 0 ; 0 ; 0]$ gives a variety which admits an obvious holomorphic map to the conic

$$
C=\left\{\left[z_{0} ; z_{1} ; z_{2}\right] \in \mathbb{C} P^{2} \mid z_{0} z_{1}=z_{2}^{2}\right\} \cong \mathbb{C} P^{1},
$$

all of whose fibres are copies of $\mathbb{C} P^{1}$. By general principles such a map is necessarily a holomorphic $\mathbb{C} P^{1}$ bundle over $\mathbb{C} P^{1}$, i.e., of the form $\mathbb{P}(\mathcal{O}(a) \oplus \mathcal{O}(0))$ for some integer $a$. The zero section (which may be identified with $C$ ) has self-intersection number 2 , so $a=2$. Thus, $\Omega_{1}$ is obtained by collapsing to a point the infinity section of $\mathbb{P}(\mathcal{O}(2) \oplus \mathcal{O}(0))$, i.e.,

$$
\Omega_{1}=\mathrm{Gr}_{2}\left(\mathbb{C}^{4}\right)^{N(2,2)} \cong \mathcal{O}(2)^{+} \cong\left(T S^{2}\right)^{+},
$$

where + indicates the Thom space. This makes precise the statement in Sect. 1 that $\Omega_{1}$ is a cone over a conic. It is also consistent with the assertion at the end of Sect. 1 that $\Omega_{1}$ is the 4-skeleton of $\Omega S U_{2} \cong S^{3}$ and it is well known that the 4-skeleton of $\Omega S^{3}$ is homotopy equivalent to $\left(T S^{2}\right)^{+}$. The cell structure of $\Omega_{1}$ itself, indicated by the diagrams above, is easy to verify using this description of $\Omega_{1}$.

We summarize below some elementary properties of the spaces $\mathrm{Gr}_{k}\left(\mathbb{C}^{n}\right)^{N}$.

Proposition 3.3. 1) $\mathrm{Gr}_{k}\left(\mathbb{C}^{n}\right)^{N} \cong \mathrm{Gr}_{n-k}\left(\mathbb{C}^{n}\right)^{N}$.

2) The inclusion $\mathrm{Gr}_{k}\left(\operatorname{Ker} N^{k}\right)^{N} \rightarrow \mathrm{Gr}_{k}\left(\mathbb{C}^{n}\right)^{N}$ is an isomorphism.

3) The inclusion $\mathrm{Gr}_{k}\left(\mathbb{C}^{m k}\right)^{N(k, \ldots, k)} \rightarrow \mathrm{Gr}_{k}\left(\mathbb{C}^{n}\right)^{N\left(n_{1}, \ldots, n_{m}\right)}$ is an isomorphism if $k \leqq n_{i}$ for $i=1, \ldots, m$.

Proof. 1) Certainly $\mathrm{Gr}_{k}\left(\mathbb{C}^{n}\right)^{N} \cong \mathrm{Gr}_{n-k}\left(\mathbb{C}^{n}\right)^{N^{t}}$. But $N$ and $N^{t}$ are similar transformations, so 1) follows. From consideration of the diagrams, 2) and 3) are obvious.

\section{Results}

Our aim is to find an explicit cell decomposition of $\operatorname{Hol}_{d}^{*}\left(S^{2}, \Omega_{k}\right)$, where $\Omega_{k}=\mathrm{Gr}_{k n}\left(\mathbb{C}^{2 k n}\right)^{N(2 k, \ldots, 2 k)}$. By Theorem 3.1, we have an explicit cell decomposition of $\Omega_{k}$. The main observation of this section is that the whole space $\operatorname{Hol}_{d}^{*}\left(S^{2}, \Omega_{k}\right)$ may in fact be constructed out of fixed point varieties of the type considered in Sect. 3 , and hence its dimension may be read off. Before proceeding, however, we need to recall some facts about holomorphic vector bundles on $S^{2}$.

The Birkhoff-Grothendieck theorem (see [Gr]) says that any holomorphic vector bundle $E$ on $S^{2}$ splits holomorphically as a direct sum of line bundles, i.e.,

$$
E \cong \mathcal{O}\left(a_{1}\right) \oplus \ldots \oplus \mathcal{O}\left(a_{n}\right),
$$


where $\mathcal{O}(a)$ is the standard line bundle of degree $a\left(a=\left(c_{1} \mathcal{O}(a)\right)\left[S^{2}\right]\right)$. This splitting is not canonical, but it is proved in $[\mathrm{Gr}]$ that there is a canonically defined sequence

$$
0 \subset E_{s_{1}} \subset E_{s_{2}} \subset \ldots \subset E_{s_{k}}=E
$$

of holomorphic subbundles, where

1) $E_{s_{l}}$ has rank $s_{i}$ and $E_{s_{i}} / E_{s_{t-1}} \cong \mathcal{O}\left(b_{i}\right) \oplus \ldots \oplus \mathcal{O}\left(b_{i}\right)$

2) $b_{1}>\ldots>b_{k}$

3) the sequences $0 \rightarrow E_{s_{2}-1} \rightarrow E_{s_{l}} \rightarrow E_{s_{l}} / E_{s_{l-1}} \rightarrow 0$ split holomorphically.

Hence, the $a_{i}$ 's are just the $b_{i}$ 's, possibly with repetitions. These subbundles may be characterized as follows: the meromorphic sections of $E$ with divisor order $\geqq i$ generate a holomorphic subbundle $F_{i} \subseteq E$, and so one has a sequence $0 \subseteq \ldots \subseteq F_{i+1} \subseteq F_{i} \subseteq \ldots \subseteq E$; the distinct subbundles of this sequence are the required ones.

If $X$ is a complex variety with a holomorphic vector bundle $E$ of rank $n$, and $f \in \operatorname{Hol}\left(S^{2}, X\right)$, then the pullback $E_{f}=f^{*} E$ splits holomorphically as $\mathcal{O}\left(a_{1}^{f}\right) \oplus \ldots \oplus \mathcal{O}\left(a_{n}^{j}\right)$ with $a_{1}^{f} \geqq \ldots \geqq a_{n}^{f}($ say $)$, so one has a "stratification" of $\operatorname{Hol}\left(S^{2}, X\right)$ given by the various splitting types. This has been studied in some depth (see, for example, $[\mathrm{AB}]$ ), but the only result we shall need is a consequence of the "semicontinuity theorem" (see $[\mathrm{Br}]$ ): if

$$
r=\min \left\{\sum_{i<j}\left(a_{i}^{f}-a_{j}^{f}\right) \mid f \in \operatorname{Hol}\left(S^{2}, X\right)\right\},
$$

then the subset of $\operatorname{Hol}\left(S^{2}, X\right)$ consisting of those $f$ for which this minimum is attained is open and dense. We shall apply this to the case where $X$ is (a subvariety of) a Grassmannian, and $E$ is the tautologous holomorphic vector bundle. The integers $a_{i}^{f}$ are then necessarily non-positive.

The following is a straightforward application of the Birkhoff-Grothendieck decomposition.

Lemma 4.1. 1) $\operatorname{dim}_{\mathbb{C}} \mathrm{Gr}_{a}\left(\mathbb{C}^{b}\right)=a(b-a)$.

2) If $d \geqq 0, \operatorname{dim}_{\mathbb{C}} \mathrm{Hol}_{d}^{*}\left(S^{2}, \operatorname{Gr}_{a}\left(\mathbb{C}^{b}\right)\right)=d b$.

Proof. 1) This follows from the equivalence $\mathrm{Gr}_{a}\left(\mathbb{C}^{b}\right) \cong U_{b} /\left(U_{a} \times U_{b-a}\right)$.

2). The tangent space to $\operatorname{Hol}_{d}\left(S^{2}, \mathrm{Gr}_{a}\left(\mathbb{C}^{b}\right)\right)$ at $f$ may be identified with the space of holomorphic sections of $f^{*} T$, where $T$ is the tangent bundle of $\operatorname{Gr}_{a}\left(\mathbb{C}^{b}\right)$. From the identification mentioned in 1$), T \cong \operatorname{Hom}\left(E, \mathbb{C}^{b} / E\right)$, where we write $\mathbb{C}^{b}$ for the trivial bundle with fibre $\mathbb{C}^{b}$. Let the Birkhoff-Grothendieck decompositions be $E \cong \mathcal{O}\left(k_{1}\right) \oplus \ldots \oplus \mathcal{C}\left(k_{a}\right)$ and $\mathbb{C}^{b} / E \cong \mathcal{O}\left(l_{1}\right) \oplus \ldots \oplus \mathcal{O}\left(l_{b-a}\right)$. Then $k_{i} \leqq 0$ for all $i$ and $\sum_{i=1}^{a} k_{i}=-d$, and $l_{j} \geqq 0$ for all $j$ and $\sum_{j=1}^{b-a} l_{j}=d$. Hence $T \cong \sum_{i, j} \mathcal{O}\left(l_{j}-k_{l}\right)$. Since $l_{j}-k_{i} \geqq 0$ for all $i, j$, the space of holomorphic sections of $f^{*} T$ has dimension $\sum_{i, j}\left(l_{j}-k_{i}+1\right)=d b+a(b-a)$, and the result follows from 1$)$.

Unfortunately, the same method does not apply when $\mathrm{Gr}_{a}\left(\mathbb{C}^{b}\right)$ is replaced by $\mathrm{Gr}_{a}\left(\mathbb{C}^{b}\right)^{N}$, so we develop a different approach which will give the dimension as a consequence of the cell decomposition. As motivation, we first make some remarks on holomorphic curves in complex projective space. 
A holomorphic map $f: S^{2} \rightarrow \mathbb{C} P^{b-1}$ of degree $d$ corresponds to a holomorphic line subbundle $E_{f} \cong \mathcal{O}(-d)$ of $\mathbb{C}^{b}$ (in the usual way) and hence to a holomorphically trivial line subbundle of $\mathbb{C}^{b} \otimes \mathcal{O}(d)$. Now, $\Gamma \mathcal{O}(d)$ may be identified naturally with $\left(\mathbb{C}^{d+1}\right)^{*}$ (or polynomials in one variable of degree $d$ ). So $f$ corresponds to a point $[T]$ in $\mathbb{P}\left(\operatorname{Hom}\left(\mathbb{C}^{d+1}, \mathbb{C}^{b}\right)\right)$. Beginning with $[T]$, if we take $1, z, z^{2}, \ldots, z^{d}$ as a basis for $\mathbb{C}^{d+1}, f$ is given explicitly in local homogeneous coordinates by $f(z)=\left[p_{1}(z) ; \ldots ; p_{b}(z)\right]$, where the coefficients of the polynomial $p_{i}$ are given by the $i$-th row of the matrix representing $T$. Conversely, a point $[T]$ arises from some $f \in \mathrm{Hol}_{d}\left(S^{2}, \mathbb{C} P^{b-1}\right)$ if and only if the polynomials $p_{1}, \ldots, p_{b}$ have no common zero in $S^{2}$, i.e., if and only if

$$
\operatorname{Ker} T \cap \operatorname{Ver}(d)=\{0\},
$$

where $\operatorname{Ver}(d)$ is the submanifold of $\mathbb{C}^{d+1}$ consisting of points which project to the "Veronese curve" $z \mapsto\left[1 ; z ; z^{2} ; \ldots ; z^{d}\right]$ in $\mathbb{C} P^{d}$.

Let $D_{d}=\left\{T \in \operatorname{Hom}\left(\mathbb{C}^{d+1}, \mathbb{C}^{b}\right) \mid \operatorname{Ker} T \cap \operatorname{Ver}(d)=\{0\}\right\}$ (a closed subset). To summarize, we have shown

$$
\operatorname{Hol}_{d}\left(S^{2}, \mathbb{C} P^{b-1}\right) \cong \mathbb{P}\left(\operatorname{Hom}\left(\mathbb{C}^{d+1}, \mathbb{C}^{b}\right)-D_{d}\right)
$$

which illustrates the classical fact that "every holomorphic curve of genus zero is the projection of a Veronese curve." We shall use this to give a second proof of Lemma 4.12 ). Unlike the first proof, this can be modified to deal with $\operatorname{Gr}_{a}\left(\mathbb{C}^{b}\right)^{N}$, and will form the basis for our main result (Theorem 4.2).

Alternative Proof of Lemma 4.12), for $d \leqq a$. The basepoint condition is $f(\infty)=\mathbb{C}^{a}$ $=\left[e_{1}, \ldots, e_{a}\right]$. We define

$$
\begin{aligned}
\operatorname{Hol}_{d}^{* *}\left(S^{2}, \operatorname{Gr}_{a}\left(\mathbb{C}^{b}\right)\right) & =\left\{f \in \operatorname{Hol}_{d}^{*}\left(S^{2}, \operatorname{Gr}_{a}\left(\mathbb{C}^{b}\right)\right) \mid E_{f}\right. \\
& \cong \mathcal{O}(-1) \oplus \ldots \oplus \mathcal{O}(-1) \oplus \mathcal{O}(0) \oplus \ldots \oplus \mathcal{O}(0)\} ;
\end{aligned}
$$

the number of summands equal to $\mathcal{O}(-1)$ is necessarily $d$. By the semi-continuity property referred to earlier, this is an open dense subset of $\operatorname{Hol}_{d}^{*}\left(S^{2}, \mathrm{Gr}_{a}\left(\mathbb{C}^{b}\right)\right)$. For each $f \in \operatorname{Hol}_{d}^{* *}\left(S^{2}, \mathrm{Gr}_{a}\left(\mathbb{C}^{b}\right)\right)$ we have a Birkhoff-Grothendieck filtration $0 \subseteq T_{f} \subseteq E_{f}\left(\subseteq S^{2} \times \mathbb{C}^{b}\right)$, where $T_{f}$ is a canonically defined, holomorphically trivial, subbundle of $E_{f}$. Now, a trivial holomorphic subbundle of $S^{2} \times \mathbb{C}^{b}$ (being determined by its holomorphic sections, which are all constant) must be of the form $S^{2} \times V$ for some subspace $V$ of $\mathbb{C}^{b}$. Hence we have a map

$$
\operatorname{Hol}_{d}^{* *}\left(S^{2}, \mathrm{Gr}_{a}\left(\mathbb{C}^{b}\right)\right) \rightarrow \mathrm{Gr}_{a-d}\left(\mathbb{C}^{b}\right), \quad f \mapsto V_{f},
$$

where $V_{f}$ is the subspace corresponding to $T_{f}$. Explicitly, we have $V_{f}=\cap\left\{f(z) \mid z \in S^{2}\right\}$. Since $f(\infty)=\mathbb{C}^{a}$, we have $V_{f} \cong \mathbb{C}^{a}$ for all $f$. We obtain a map

$$
\pi: \operatorname{Hol}_{d}^{* *}\left(S^{2}, \operatorname{Gr}_{a}\left(\mathbb{C}^{b}\right)\right) \rightarrow \operatorname{Gr}_{a-d}\left(\mathbb{C}^{a}\right),
$$

which is obviously surjective. In fact $\pi$ is a holomorphic fibre bundle, whose fibre over $X \subseteq \mathbb{C}^{a}$ is

$$
\pi^{-1}(X) \cong \operatorname{Hol}_{d}^{* *}\left(S^{2}, \mathrm{Gr}_{d}\left(\left(\mathbb{C}^{a} / X\right) \oplus\left(\mathbb{C}^{a}\right)^{\perp}\right)\right) .
$$

Here, the basepoint condition is $g(\infty)=\mathbb{C}^{a} / X$, and $E_{g} \cong \mathcal{O}(-1) \oplus \ldots \oplus \mathcal{O}(-1)$. We shall now calculate the dimensions of the base and fibre of $\pi$. 
Bearing in mind the remarks above on holomorphic curves in complex projective space, we can see that the fibre $\pi^{-1}(X)$ may be identified with a subset of the Grassmannian $\mathrm{Gr}_{d}\left(\mathbb{C}^{2 d+b-a}\right)$. For a map

$$
g \in \operatorname{Hol}_{d}^{* *}\left(S^{2}, \operatorname{Gr}_{d}\left(\left(\mathbb{C}^{a} / X\right) \oplus\left(\mathbb{C}^{a}\right)^{\perp}\right)\right)
$$

corresponds to a holomorphically trivial subbundle, of rank $d$, of the bundle

$$
\left[\left(\mathbb{C}^{a} / X \oplus\left(\mathbb{C}^{a}\right)^{\perp}\right] \otimes \mathcal{O}(1) .\right.
$$

To identify the $d$-planes which arise in this way, we introduce some notation: $s_{1}, s_{2}$ is a basis of $\mathbb{C}^{2}, f_{1}, \ldots, f_{d}$ is a basis of $\mathbb{C}^{a} / X$, and $e_{a+1}, \ldots, e_{b}$ is the standard basis of $\left(\mathbb{C}^{a}\right)^{\perp}$. The condition that $W$ defines a holomorphic map of degree $d$ such that

$$
E_{g} \cong \mathcal{O}(-1) \oplus \ldots \oplus \mathcal{O}(-1)
$$

is that a basis $T_{1}, \ldots, T_{d}$ of $W$ can be chosen such that $\operatorname{Ker} T_{i} \cap \operatorname{Ver}(1)=\{0\}$ for all $i$, i.e., such that $\operatorname{Ker} T \cap \operatorname{Ver}(1)=\{0\}$ for all $T \in W$. The map is then given by

$$
g(z)=\left[T_{1}\left(s_{1}+z s_{2}\right), \ldots, T_{d}\left(s_{1}+z s_{2}\right)\right],
$$

so the basepoint condition $g(\infty)=\mathbb{C}^{a} / X$ is that $T\left(s_{1}\right) \in \mathbb{C}^{a} / X$ for all $T \in W$. Hence $\pi^{-1}(X)$ may be identified with the open subset $\mathrm{Gr}_{d}\left(\mathbb{C}^{2 d+b-a}-D\right)$ of $\mathrm{Gr}_{d}\left(\mathbb{C}^{2 d+b-a}\right)$, where $\mathbb{C}^{2 d+b-a}$ has basis consisting of

$$
s_{1}^{*} \otimes f_{1}, \ldots, s_{1}^{*} \otimes f_{d} ; \quad s_{2}^{*} \otimes f_{1}, \ldots, s_{2}^{*} \otimes f_{d} ; \quad s_{2}^{*} \otimes e_{a+1}, \ldots, s_{2}^{*} \otimes e_{b} ;
$$

and $D$ consists of those points

$$
T \in \mathbb{C}^{2 d+b+a} \cong \operatorname{Hom}\left(\mathbb{C}^{2},\left(\mathbb{C}^{a} / X\right) \oplus\left(\mathbb{C}^{a}\right)^{\perp}\right)
$$

which are not of maximal rank. Hence the fibre and base of $\pi$ have dimensions $d(d+b-a), d(a-d)$ respectively by 4.11$)$ and so the total space has (complex) dimension $d b$.

Our main result is a description of the restriction, $\pi_{N}$, of

$$
\pi: \operatorname{Hol}_{d}^{* *}\left(S^{2}, \mathrm{Gr}_{k n}\left(\mathbb{C}^{2 k n}\right)\right) \rightarrow \mathrm{Gr}_{k n-d}\left(\mathbb{C}^{k n}\right)
$$

to $\operatorname{Hol}_{d}^{* *}\left(S^{2}, \Omega_{k}\right)$.

Theorem 4.2. Assume $0 \leqq d \leqq k n$. Then,

1) The image of $\pi_{N}$ is $\pi_{N}\left(\operatorname{Hol}_{d}^{* *}\left(S^{2}, \Omega_{k}\right)\right)=\mathrm{Gr}_{k n-d}\left(\mathbb{C}^{k n}\right)^{N(k, \ldots, k)}$.

2) If $X \in \mathrm{Gr}_{k n-d}\left(\mathbb{C}^{k n}\right)^{N(k, \ldots, k)}$, the fibre $\pi_{N}^{-1}(X)$ is isomorphic to

$$
\mathrm{Gr}_{d}\left(\mathbb{C}^{2 d+k n}-D\right)^{N\left(k+k_{1}^{X}, \ldots, k+k_{n}^{X}, k_{1}^{X}, \ldots, k_{n}^{X}\right)}
$$

for some $k_{1}^{X}, \ldots, k_{n}^{X} \geqq 0$ with $k_{1}^{X}+\ldots+k_{n}^{X}=d$, where $D$ is the closed subset of $\mathbb{C}^{2 d+k n}$ defined in the previcus paragraph.

Proof. Take $f \in \operatorname{Hol}_{d}^{* *}\left(S^{2}, \operatorname{Gr}_{k n}\left(\mathbb{C}^{2 k n}\right)\right)$. This defines a $(k n-d)$-plane

$$
V_{f}=\pi(f) \in \mathrm{Gr}_{k n-d}\left(\mathbb{C}^{2 k n}\right),
$$

and a map

$$
g \in \operatorname{Hol}_{d}^{* *}\left(S^{2}, \operatorname{Gr}_{d}\left(\left(\mathbb{C}^{k n} / V_{f}\right) \oplus\left(\mathbb{C}^{k n}\right)^{\perp}\right)\right)
$$

$\left[\right.$ namely, $\left.g(z)=f(z) / V_{f} \oplus\left(\mathbb{C}^{k n}\right)^{\perp}\right]$. 
Claim. $N f(z) \subseteq f(z)$ for all $z \in S^{2}$ [i.e., $f\left(S^{2}\right) \subseteq \Omega_{k}$ ] if and only if $N V_{f} \subseteq V_{f}$ and $N g(z) \subseteq g(z)$ for all $z \in S^{2}$.

This is obvious since $V_{f}=\cap\left\{f(z) \mid z \in S^{2}\right\}$. The nilpotent linear transformations induced by $N$ on $\mathbb{C}^{k n}$ and on $\left(\mathbb{C}^{k n} / V_{f}\right) \oplus\left(\mathbb{C}^{k n}\right)^{\perp}$ will also be denoted $N$ when this is unlikely to lead to confusion. Thus, on $\mathbb{C}^{k n}, N=N(k, \ldots, k)$, so the image of $\pi_{N}$ is $\mathrm{Gr}_{k n-d}\left(\mathbb{C}^{k n}\right)^{N(k, \ldots, k)}$.

To identify the fibre of $\pi_{N}$ over $V_{f}$ we need to find the type of $N$ on $\mathbb{C}^{d+k n}$ $\cong\left(\mathbb{C}^{k n} / V_{f}\right) \oplus\left(\mathbb{C}^{k n}\right)^{\perp}$

Lemma 4.3. Let $N=N(m, \ldots, m)$ on $\mathbb{C}^{l m}$, so that $\operatorname{Ker} N^{i}=\mathbb{C}^{l i}$ for $i=1, \ldots, m$. Let $X \in \mathrm{Gr}_{k}\left(\mathbb{C}^{l m}\right)$ with $N X \subseteq X$. Then

1) $N=N\left(a_{1}, \ldots, a_{l}\right)$ on $X$, for some $a_{1}, \ldots, a_{l} \leqq m$.

2) $N=N\left(m-a_{1}, \ldots, m-a_{l}\right)$ on $\mathbb{C}^{l m} / X$.

Moreover, if $X \subseteq \mathbb{C}^{l i}$, then $a_{1}, \ldots, a_{l} \leqq i$.

This will be proved later. It follows that $N=N\left(k_{1}, \ldots, k_{n}\right)$ on $\mathbb{C}^{k n} / X$ and that $N=N\left(k+k_{1}, \ldots, k+k_{n}\right)$ on $\left(\mathbb{C}^{k n} / X\right) \oplus\left(\mathbb{C}^{k n}\right)^{\perp}$, for some $k_{1}, \ldots, k_{n}(\geqq 0)$ with $k_{1}+\ldots+k_{n}=d$. Hence $N=N\left(k+k_{1}, \ldots, k+k_{n}, k_{1}, \ldots, k_{n}\right)$ on

$$
\mathbb{C}^{2 d+k n} \cong\left[\left(\mathbb{C}^{k n} / X\right) \oplus\left(\mathbb{C}^{k n}\right)^{\perp}\right] \oplus\left[\mathbb{C}^{k n} / X\right]
$$

This completes the proof of Theorem 4.2.

Proof of Lemma 4.3. If $X \in \mathrm{Gr}_{k}\left(\mathbb{C}^{l m}\right)^{N}$, then $X$ belongs to a cell of $\mathrm{Gr}_{k}\left(\mathbb{C}^{l m}\right)^{N}$ corresponding to an $l \times m$ diagram ( $l$ rows and $m$ columns) of the form

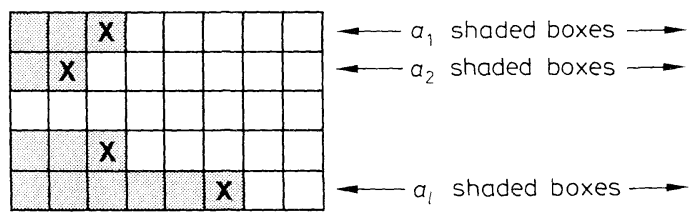

(see Theorem 3.1). Hence $N=N\left(a_{1}, \ldots, a_{l}\right)$ on $X$, and 1) is proved. To prove 2), we begin by choosing a basis of $X$ of the form $x_{1}, N x_{1}, \ldots, N^{a_{1}-1} x_{1} ; \ldots ; x_{l}, N x_{l}, \ldots$, $N^{a_{l}-1} x_{l}$, which is possible by 1$)$. Since $\operatorname{Ker} N^{i}=\mathbb{C}^{l i}=\operatorname{Im} N^{m-i}$, we may choose $y_{1}, \ldots, y_{l} \in \mathbb{C}^{l m}$ with $N^{m-k} y_{1}=x_{1}, \ldots, N^{m-k_{l}} y_{l}=x_{l}$. We claim that $y_{1}, N y_{1}, \ldots$, $N^{m-1} y_{1} ; \ldots ; y_{l}, N y_{l}, \ldots, N^{m-1} y_{l}$ is a basis for $\mathbb{C}^{l m}$. If

$$
\sum_{i=0}^{m} \alpha_{i 1} N^{i} y_{l}+\ldots+\sum_{i=0}^{m} \alpha_{i l} N^{i} y_{l}=0
$$

then application of $N^{m-1}$ gives $\alpha_{01} N^{m-1} y_{l}+\ldots+\alpha_{0 l} N^{m-l} y_{l}=0$, contradicting linear independence of the chosen basis for $X$, unless $\alpha_{01}=\ldots=\alpha_{0 l}=0$. Application of $N^{m-j}$ for $j=2, \ldots, m$ shows similarly that $\alpha_{j 1}=\ldots=\alpha_{j l}=0$ for all $j$, so the claim is proved. Using the quotient basis for $\mathbb{C}^{l m} / X$ gives 2). Finally, if $X \subseteq \mathbb{C}^{l i}$, then the observation used in the proof of 1) shows that $a_{1}, \ldots, a_{l} \leqq i$, since all shaded boxes must appear in the first $i$ columns of the diagram.

We can now prove Corollary 1.4 of the introduction. 
First, $\operatorname{dim} \operatorname{Hol}_{d}^{*}\left(S^{2}, \Omega_{k}\right)=\operatorname{dim} \operatorname{Hol}_{d}^{* *}\left(S^{2}, \Omega_{k}\right)$ since the second space is an open dense subspace of the first. To use Theorem 4.2, we shall calculate the dimensions of the base and of all fibres of $\pi_{N}$. The base is $\mathrm{Gr}_{k n-d}\left(\mathbb{C}^{k n}\right)^{N(k, \ldots, k)}$ $\cong \mathrm{Gr}_{d}\left(\mathbb{C}^{k n}\right)^{N(k, \ldots, k)}$ (by 1) of Proposition 3.3), and by Theorem 3.1 this has a cell decomposition with cells corresponding to $n \times k$ diagrams of the form:

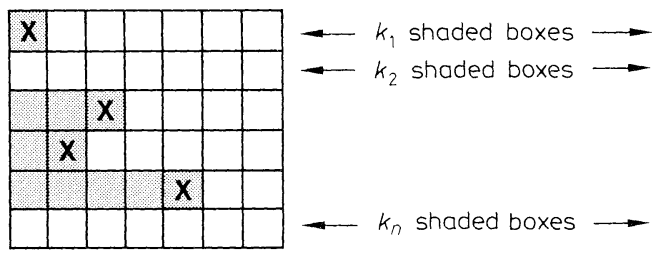

The total number of shaded boxes is $k_{1}+\ldots+k_{n}=d$. The largest dimension of such a cell (e.g., for $k_{1}=\ldots=k_{n-1}=0, k_{n}=d$ ) is $2(2 n-1) d$, so this is the dimension of the base. For any $X \in \mathrm{Gr}_{k n-d}\left(\mathbb{C}^{k n}\right)^{N(k, \ldots, k)}$ which is contained in the cell corresponding to such a diagram, we have $N=N\left(k_{1}, \ldots, k_{n}\right)$ on $\mathbb{C}^{k n} / X$. By Theorem 4.2 , the fibre $\pi_{N}^{-1}(X)$ over any such point $X$ has the same dimension as

$$
\mathrm{Gr}_{d}\left(\mathbb{C}^{2 d+k n}\right)^{N\left(k+k_{1}, \ldots, k+k_{n}, k_{1}, \ldots, k_{n}\right)} \text {. }
$$

To find the dimension of this, using Theorem 3.1, we must shade $d$ boxes of the diagram

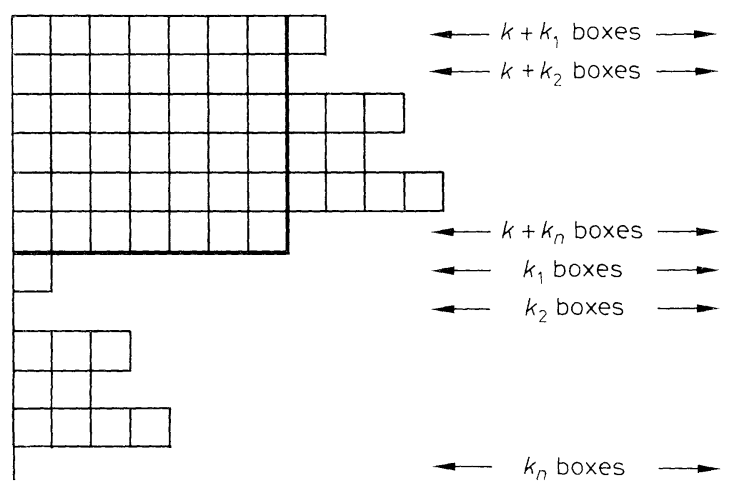

in such a way that the dimension of the corresponding cell is maximized. It is easy to see that this is achieved by (for example) shading the last $n$ rows. The dimension is then $n d$. As this is independent of $k_{1}, \ldots, k_{n}$ all fibres of $\pi_{N}$ have the same dimension $n d$, so $\operatorname{dim}_{\mathbb{C}} \mathrm{Hol}_{d}^{* *}\left(S^{2}, \Omega_{k}\right)=n d+(n-1) d=(2 n-1) d$.

Remarks. 1) Consideration of the last proof shows that in fact $\operatorname{Hol}_{d}^{* *}\left(S^{2}, \Omega_{d}\right)$ $=\operatorname{Hol}_{d}^{* *}\left(S^{2}, \Omega_{k}\right)$ for all $k \geqq d$. [The base of $\pi_{N}$ is $\mathrm{Gr}_{k n-d}\left(\mathbb{C}^{k n}\right)^{N(k \ldots, k)}$ $\cong \mathrm{Gr}_{d}\left(\mathbb{C}^{k n}\right)^{N(k, \ldots, k)} \cong \mathrm{Gr}_{d}\left(\mathbb{C}^{d n}\right)^{N(d, \ldots, d)}$ by 1) and 3) of Proposition 3.3. A similar reduction may be made for the fibre.]

2) We have dealt only with the open dense subspace $\operatorname{Hol}_{d}^{* *}\left(S^{2}, \Omega_{k}\right)$ of $\operatorname{Hol}_{d}^{*}\left(S^{2}, \Omega_{k}\right)$, but in principle our methods of constructing the bundle $\pi$ applies to any other stratum. The calculations would be more complicated, however.

Proof of Corollary 1.4. The cell decomposition follows from Theorems 3.1 and 4.2. By definition, $\operatorname{dim} \operatorname{Hol}_{d}\left(S^{2}, \Omega_{\mathrm{alg}} S U_{n}\right)=\lim _{k \rightarrow \infty} \operatorname{dim} \mathrm{Hol}_{d}\left(S^{2}, \Omega_{k}\right)$, and this is $2(2 n-1) d$ by Corollary 4.4 . 
We conclude with some remarks on the case $d=1, n=2$. Here we have

$$
\begin{aligned}
\operatorname{Hol}_{1}^{*}\left(S^{2}, \Omega_{k}\right) & =\operatorname{Hol}_{1}^{* *}\left(S^{2}, \Omega_{k}\right) & & \text { (obvious) } \\
& =\operatorname{Hol}_{1}^{* *}\left(S^{2}, \Omega_{1}\right) & & \text { (see above) }
\end{aligned}
$$

and this has dimension 6 by the proof of 1.4. It is possible to obtain the dimension of $\operatorname{Hol}_{1}^{*}\left(S^{2}, \Omega_{1}\right)$ by direct arguments, as follows. First, as in the introduction, we may use the explicit description of $\Omega_{1}$ as $\mathcal{O}(2)^{+}$(see Example 3.2), from which it follows that $\operatorname{Hol}_{1}^{*}\left(S^{2}, \Omega_{1}\right)$ is just the principal Aff $(\mathbb{C})$ bundle associated to $\mathcal{O}(2)$. An alternative, more elementary, argument proceeds by a modification of the method of Theorem 4.2. We have a map

$$
\pi: \operatorname{Hol}_{1}^{*}\left(S^{2}, \mathrm{Gr}_{2 k}\left(\mathbb{C}^{4 k}\right)\right) \rightarrow F(2 k-1,2 k+1,4 k)
$$

obtained by defining $\pi(f)$ to the flag

$$
0 \subset \cap\left\{f(z) \mid z \in S^{2}\right\} \subset \bigcup\left\{f(z) \mid z \in S^{2}\right\} \subset \mathbb{C}^{4 k}
$$

Note that $\cup\left\{f(z) \mid z \in S^{2}\right\}$ is in fact a linear subspace of $\mathbb{C}^{4 k}$ since $f$ has degree 1 , so $\pi$ is well defined. The image of this map is

$$
\left\{0 \subset X \subset Y \subset \mathbb{C}^{4 k} \mid X \subset \mathbb{C}^{2 k} \subset Y\right\} \cong \mathbb{C} P^{2 k-1} \times \mathbb{C} P^{2 k-1},
$$

and the fibre over $X \subset Y \subset \mathbb{C}^{4 k}$ is isomorphic to

$$
\operatorname{Hol}_{1}^{*}\left(S^{2}, \mathbb{P}(Y / X)\right) \cong \operatorname{Aff}(\mathbb{C}) .
$$

To describe the restriction $\pi_{N}$ of $\pi$ to $\operatorname{Hol}_{1}^{*}\left(S^{2}, \Omega_{k}\right)$, we note that if $N f(z) \subseteq f(z)$ for all $z \in S^{2}$, so that $N X \subseteq X$ and $N Y \subseteq Y$, then $N=0$ on $Y / X$ and in fact $N Y=X$. (To see this, observe that if $y \in Y$ then the linear $\operatorname{span}[y, X]$ is $f(z)$ for some $z \in S^{2}$, so $N[y, X]=N f(z) \subseteq f(z)=[y, X]$, i.e., $N$ acts diagonally on $Y / X$. But $N$ is nilpotent on $Y / X$, so it must be zero. This shows $N Y \subseteq X$, but since $\operatorname{dim}_{\mathbb{C}} N Y$ $\geqq \operatorname{dim}_{\mathbb{C}} Y-2$ because of the form of $N$, we must have $N Y=X$.) Hence $\pi_{N}$ is obtained from $\pi$ simply by restricting the base to $\left\{0 \subset X \subset Y \subset \mathbb{C}^{4 k} \mid X \subset \mathbb{C}^{2 k} \subset Y\right.$, $N Y=X\} \cong \mathbb{P}\left(\mathbb{C}^{2 k+2} / \mathbb{C}^{2 k}\right) \cong \mathbb{C} P^{1}\left(\cong S^{2}\right)$. Again this exhibits $\operatorname{Hol}_{1}^{*}\left(S^{2}, \Omega_{\mathrm{alg}} S U_{2}\right)$ as an $\operatorname{Aff}(\mathbb{C})$-bundle over $\mathbb{C} P^{1}$. From either of these descriptions, one sees that $\operatorname{Hol}_{1}^{*}\left(S^{2}, \Omega_{\text {alg }} S U_{2}\right)$ is complex analytically equivalent to $P S L_{2}(\mathbb{C})$.

Acknowledgements. The authors wish to thank the Mathematical Sciences Research Institute, Berkeley, where most of this work was carried out, and H. Flenner, D. S. Freed, F. C. Kirwan, S. Kumar, and G. B. Segal for useful conversations. The first author is grateful for the support of the Humboldt Foundation and the Max-Planck-Institut für Mathematik, Bonn.

\section{References}

[AB] Atiyah, M.F., Bott, R.: The Yang-Mills equations over Riemann surfaces. Phil. Trans. R. Soc. Lond. A 308, 523-615 (1982)

[AHS] Atiyah, M.F., Hitchin, N.J., Singer, I.M.: Self-duality in four dimensional Riemannian geometry. Proc. R. Soc. Lond. A 362, 425-461 (1978)

[AJ] Atiyah, M.F., Jones, J.D.S.: Topological aspects of Yang-Mills theory. Commun. Math. Phys. 61, 97-118 
[At] M.F. Atiyah: Instantons in two and four dimensions. Commun. Math. Phys. 93, 437-451 (1984)

[BM] Boyer, C., Mann, B.: Homology operations on instantons (to appear)

[Bo] Borho, W.: Nilpotent orbits, primitive ideals, and characteristic classes. Proc. of the International Congress of Mathematicians 1986 (to appear)

[Br] Brieskorn, E.: Über holomorphe $P_{n}$-Bündel über $P_{1}$. Math. Ann. 157, 343-357 (1965)

[FU] Freed, D.S., Uhlenbeck, K.K.: Instantons and four-manifolds. M.S.R.I. Publications, Vol. 4. Berlin, Heidelberg, New York: Springer 1984

[Gr] Grothendieck, A.: Sur la classification des fibres holomorphes sur la sphere de Riemann. Am. J. Math. 79, 121-138 (1957)

[Gu] Guest, M.A.: Topology of the space of absolute minima of the energy functional. Am. J. Math. 106, 21-42 (1984)

[Gv] Graveson, J.: On the topology of spaces of holomorphic maps. D. Phil. thesis, Oxford, 1987

[HS] Hotta, R., Shimomura, N.: The fixed point subvarieties of unipotent transformations on generalized flag varieties and the Green functions. Math. Ann. 241, 193-208 (1979)

[Ki] Kirwan, F.: On spaces of maps from Riemann surfaces to Grassmannians and applications to the cohomology of moduli of vector bundles. Arkiv. Math. 24, 221-275 (1986)

[Pr] Pressley, A.N.: Decompositions of the space of loops on a Lie group. Topology 19,65-79 (1980)

[PS] Pressley, A.N., Segal, G.B.: Loop groups. Oxford: Oxford University Press 1986

[Se] Segal, G.B.: The topology of spaces of rational functions. Acta Math. 143, 39-72 (1979)

[Sh] Shimomura, N.: A theorem on the fixed point set of a unipotent transformation. J. Math. Soc. Jpn. 32, 55-64 (1980)

[Ta] Taubes, C.H.: The stable topology of self-dual moduli spaces. Preprint, Harvard, 1986

[Uh] Uhlenbeck, K.K.: Harmonic maps into Lie groups (classical solutions of the chiral model). Preprint, Chicago, 1985

[ZM] Zakharov, V.E., Mikhailov, A.V.: Relativistically invariant two-dimensional models of field theory which are integrable by means of the inverse scattering problem method. Sov. Phys. JETP 47, 1017-1027 (1978)

[ZS] Zakharov, V.E., Shabat, A.B.: Integration of nonlinear equations of mathematical physics by the method of inverse scattering. II. Funct. Anal. Appl. 13, 166-174 (1979)

Communicated by L. Alvarez-Gaumé

Received February 26, 1988

Note added in proof. For $G=S U_{2}$, Boyer and Mann have used Dyer-Lashoff operations to detect homology of $\mathscr{I}_{d} / \mathscr{G}_{*}$ up to dimension (approximately) $\frac{3}{4} \operatorname{dim} \mathscr{I}_{d} / \mathscr{G}_{*}$. (A classifying space for instantons, preprint, Clarkson University, 1988.) The similarity with our result, that $\operatorname{dim} \mathrm{Hol}_{d}^{*}\left(S^{2}, \Omega_{\text {alg }} S U_{2}\right)=\frac{3}{4} \operatorname{dim} \mathrm{Hol}_{d}^{*}\left(S^{2}, \Omega S U_{2}\right)$, is striking, and lends further credence to the Atiyah-Jones conjecture. 
